Cahiers québécois de démographie

Revue internationale d'étude des populations

WHITBY, A. 2020. The Sum of the People: How the Census Has

Shaped Nations, from the Ancient World to the Modern Age.

Basic Books, 338 p.

\title{
Quentin Wallut
}

Volume 48, numéro 2, automne 2019

URI : https://id.erudit.org/iderudit/1074185ar

DOI : https://doi.org/10.7202/1074185ar

Aller au sommaire du numéro

Éditeur(s)

Association des démographes du Québec

ISSN

1705-1495 (numérique)

Découvrir la revue

Citer ce compte rendu

Wallut, Q. (2019). Compte rendu de [WHITBY, A. 2020. The Sum of the People: How the Census Has Shaped Nations, from the Ancient World to the Modern Age. Basic Books, 338 p.] Cahiers québécois de démographie, 48(2), 235-239.

https://doi.org/10.7202/1074185ar d'utilisation que vous pouvez consulter en ligne. 


\section{Cahiers québécois de démographie}

Vol. 48, n² 2, automne 2019, p. 235-239

\section{Note de lecture}

WHITBY, A. 2020. The Sum of the People: How the Census Has Shaped Nations, from the Ancient World to the Modern Age. Basic Books, 338 p.

$\mathrm{L}$ es livres proposant une histoire du recensement de la population, notamment à l'échelle nationale, ne sont pas rares : leur objet a depuis longtemps été identifié comme un instrument important et très particulier. Certains travaux récents sur ce sujet ont d'ailleurs été remarqués (Emigh et collab. 2016a ; 2016b; Thorvaldsen, 2017). À l'inverse, les livres d'histoire du recensement s'adressant explicitement à un public généraliste, bien au-delà des seuls cercles académiques, sont sensiblement moins courants. C'est l'une des raisons pour lesquelles le livre d'Andrew Whitby est très bienvenu. Économiste et quantitativiste de formation, l'auteur s'essaie ici à un travail assez éloigné de son domaine d'origine et fait œuvre d'historien. Le résultat final est des plus convaincants!

Le prologue du livre est un modèle du genre et un aperçu de l'approche adoptée par Whitby. Il commence par raconter son voyage en Palestine en 2017, et à travers une poignée d'anecdotes pleines de vie, tel qu'une randonnée dans les collines rocheuses au-dessus de Bethléem ou encore ses entretiens avec des responsables du recensement palestinien, il établit les différentes dimensions historiques, politiques, identitaires et techniques du recensement. Pour étudier le recensement, il y a peu de cas plus pertinents que ceux d'Israël et de la Palestine. On trouve distillés dans ces deux cas tous les éléments qui rendent cette source de données si particulière. Pour commencer, l'un des recensements les plus fameux de l'histoire en Occident est sans doute celui qui amena Joseph et Marie à se rendre à Bethléem, tandis que l'histoire du premier recensement israélien en 1948 a été identifiée comme un cas d'école sur le rôle du recensement dans la construction d'un État et d'une population nationale (Leibler, 2004; Leibler et Breslau 2005). De même, pour les agents du recensement palestinien, mettre en place une telle collecte est un défi technique et politique important. Outre d'innombrables difficultés pratiques, le recensement a une incidence géopolitique pour Israël et la Palestine lorsqu'il s'agit de dénombrer les habitants de certains territoires disputés. La nature du recensement, à la fois outil administratif et instrument politique, est ainsi présentée dans toute son ambigüité.

C'est par ce prologue engageant par sa richesse et sa complexité que Whitby amorce son histoire des recensements, de ses débuts impossibles à déterminer (Chapitre 1: "The Book of Number ») jusqu'à ses évolutions les plus récentes. Le tableau ainsi brossé suit une évolution chronologique soigneusement construite. La période et l'espace considérés sont vastes, et l'auteur doit parfois traverser les époques un peu rapidement, mais les enjeux successifs du recensement sont l'objet de fréquents rappels et permettent de ne jamais perdre de vue la chronologie. Le récit n'est 
cependant pas strictement linéaire. En effet, pendant longtemps, des formes très variées de dénombrements ont existé à travers le monde, reposant sur des prémisses et des pratiques bien différentes. Le lecteur est transporté à travers le monde et le temps pour observer différentes histoires et méthodes. Une des grandes qualités de l'auteur est de parvenir à tenir ensemble l'irréductible variété des pratiques nationales et le principe commun du dénombrement d'une population. La substance de la thèse présentée est que le recensement ne peut se penser hors de son contexte scientifique et politique. Whitby veut aussi éclairer les implications plus larges de ces dénombrements. Progressivement, le récit réduit son champ et se concentre de plus en plus sur les pratiques européennes et américaines. Le modèle européen du recensement se met en place au milieu du XIX ${ }^{\mathrm{e}}$, fondé sur des avancées conjointes de la science, des méthodes statistiques et du champ d'action de l'État, et devient progressivement la référence jusqu'aujourd'hui. Il s'agit d'ailleurs du modèle proposé par l'ONU, et celui utilisé dans la plupart des pays. Ce n'est que récemment que des formes concurrentes émergent, fondées sur l'utilisation de registres, notamment des registres centralisés de la population et des techniques d'échantillonnage perfectionnées.

Les chapitres 2 et 3 ( "Political Arithmetics » et "A Punch Photograph ») couvrent l'histoire du recensement du $\mathrm{xvII}^{\mathrm{e}}$ aux débuts $\mathrm{du} \mathrm{xx}^{\mathrm{e}}$ siècle et racontent les conditions scientifiques, politiques et techniques entourant la naissance du recensement moderne. L'arithmétique politique nait en Angleterre au xvII ${ }^{\mathrm{e}}$ siècle, issue autant de la révolution astronomique (Copernic, Kepler, Newton), que des bouleversements politiques (guerre civile, création du Commonwealth, la Restauration puis la Glorieuse Révolution). Whitby prend soin de montrer que l'émergence des concepts de la démographie et du recensement ne peut être détachée de ce bouillonnement exceptionnel. À partir du XVIII ${ }^{\mathrm{e}}$ siècle, certains recensements commencent à se rapprocher de leur forme moderne, mais visent encore principalement à évaluer les ressources militaires et fiscales disponibles : c'est ce qui rend révolutionnaire le recensement américain et le projet politique de représentation qui lui est associé. Le troisième chapitre est quant à lui articulé autour de questions plus matérielles : en 1890, le recensement se mécanise grâce aux machines Hollerith ${ }^{1}$. Se nourrissant du passage progressif et crucial au recensement nominatif, cette évolution technique a un impact significatif sur le recensement, dès lors moins contraint dans son contenu grâce à la puissance de traitement offerte par les machines. Le $\mathrm{xIx}^{\mathrm{e}}$ siècle voit émerger un recensement « moderne » dont la mission est de renseigner les fonctions redistributives de l'État.

Le chapitre 4 ( «Paper people») porte sur les dangers du recensement, et plus généralement des registres de population. Dans un chapitre saisissant, l'auteur montre comment des bureaucrates néerlandais dévoués, mais pris d'un « rêve d'omniscience » assez courant à l'époque, ont vu leur travail soigné et systématique utilisé par l'occupant nazi, sans que ces derniers rencontrent une grande opposition. Cette analyse n'est pas nouvelle : Seltzer et Anderson (2001) en particulier ont bien montré l'usage systématique des données statistiques par les nazis (et par d'autres régimes, autoritaires ou non) pour identifier et opprimer une partie de leur population. La qualité propre de ce livre est sa force évocatrice. Il est d'autant plus regrettable que l'auteur ne revienne pas sur ces idées dans sa conclusion. La question est en effet intéressante, notamment en France, où la

1. Herman Hollerith (1860-1929) est l'un des fondateurs de l'entreprise qui deviendra IBM en 1911. 
polémique sur les statistiques ethniques ne cesse de se renouveler, mais aussi pour revenir au cas néerlandais. Depuis les années 1980, le pays a de nouveau mis en place un registre de population universel, en raison même de l'opposition d'une partie de la population au recensement classique! Pareillement, les « rêves d'omniscience » que l'on trouve chez nombre de bureaucrates entre la fin du xix ${ }^{\mathrm{e}}$ siècle et la Seconde Guerre mondiale (p. 146) auraient pu être revisités avec profit, considérant la fascination contemporaine pour les «big data ». C'est un sujet sur lequel il ne s'attarde guère, même dans ses derniers chapitres.

Les trois derniers chapitres considèrent les développements du recensement depuis la fin de la Seconde Guerre mondiale, en interrogeant certaines des grandes questions techniques et politiques auxquelles le recensement est confronté : l'émergence, puis le déclin de la croissance explosive de la population mondiale comme enjeu global entre les années 1960 et 1980 (Chapitre 5 : «A World Census ») ; le sous-dénombrement comme problème majeur du recensement depuis la mise au point d'outil permettant sa mesure (Chapitre 6: "The Uncounted») et enfin, les problèmes les plus récents du recensement depuis les années 2000 (Chapitre 7 : «The Transparent Citizen »). Le recensement est alors bien en place, mais le monde change à un rythme accéléré avec la fin des empires coloniaux, de la Guerre froide et l'accélération de la mondialisation. En parallèle, de nouveaux outils statistiques et technologiques sont développés pour améliorer le recensement, et corriger ses défauts structurels. Whitby évoque aussi l'émergence d'une critique postmoderniste plus fondamentale des statistiques, qui fragilise l'autorité des statisticiens. S’il dénonce certains excès de ces approches, il les utilise abondamment.

Brillant et captivant, procurant une lecture aisée et stimulante, ce livre peut cependant faire l'objet de quelques critiques qui ne remettent pas en cause ses qualités générales. Pour commencer, une remarque s'impose quant au sous-titre de l'ouvrage: how the census has shaped nations. Contrairement à ce que cette formule pourrait laisser présager, ce livre n'est pas un approfondissement de l'approche développée par Anderson dans son ouvrage de référence Imagined Communities (Anderson, 1983). Si Whitby a clairement intégré cette thèse et développe des idées similaires à de multiples endroits dans son texte, il ne prétend pas établir un récit de la manière dont la notion de nation et l'outil du recensement se sont coconstruits depuis le $\mathrm{xviII}^{\mathrm{e}}$ siècle. Il va ainsi passer un moment plus long sur l'idée de mécanisation de l'économie américaine à la fin du xix ${ }^{\mathrm{e}}$ siècle que sur celle de nation. Son propos ne laisse cependant pas place au doute : le recensement donne à voir la nation comme aucun autre instrument ne le peut avant lui, et cela avec une efficacité accrue à mesure que la numératie se répand au sein de la population. Il s'agit pour reprendre la formule de Paul Schor, d'un "miroir de la nation" (Schor, 2009). Le chapitre portant sur le sousdénombrement commence ainsi par montrer à quel point la question est brûlante dans une nation fragile comme l'Afrique du Sud après l'Apartheid. Sa lecture du phénomène est essentiellement top-down : sans nier que le recensement repose sur une coopération intensive avec la population, pour Whitby, l'État demeure l'acteur principal. En cela, il ne semble pas souscrire pleinement aux conclusions des travaux récents d'Emigh et collaborateurs (2016a; 2016b) (qu'il cite cependant abondamment).

De manière générale, le lecteur académique pourra déplorer le manque de conceptualisation $a$ priori. Ainsi, Whitby ne va pas chercher à offrir une définition particulièrement rigoureuse du recensement. Les termes census et enumeration sont ainsi utilisés presque indifféremment, et la 
place donnée aux statistiques démographiques en général tend à diluer les spécificités du recensement comme statistique officielle, dont le contenu va bien au-delà d'un simple dénombrement. Si cela permet d'épargner au lecteur non spécialiste des discussions trop techniques, les réflexions détaillées de Thorvaldsen ou Ventresca sur l'émergence du recensement moderne et ses critères de définition ne sont pas sans intérêt pour éclaircir les rapports essentiels entre ce phénomène et la naissance des États nations modernes aux xviII et xix ${ }^{\mathrm{e}}$ siècles (Thorvaldsen, 2017; Ventresca, 1995). La mise sur le même plan conceptuel des recensements bibliques et des recensements américains contemporains tend sans doute à trop rapprocher des pratiques qui ne partagent en réalité que peu de choses. Par ailleurs, on pourra remarquer un certain déséquilibre dans la structure même du livre : la période postérieure aux années 1990, donc il y a tout de même 30 ans, reçoit un traitement plus rapide, notamment en termes d'analyse. La chose est particulièrement sensible dans le dernier chapitre, très riche, mais qui se disperse un peu et qui passe rapidement sur nombre d'enjeux cruciaux. En particulier, les transformations fondamentales du recensement depuis une vingtaine d'années ne sont pas présentées avec la même clarté synthétique dont il a su faire preuve dans d'autres parties du livre.

Le livre a aussi les défauts de ses qualités. Tous les développements du recensement sont soigneusement replacés dans leur contexte technique, politique et intellectuel, mais parfois au point que l'exposition de ce contexte fait perdre de vue l'objet premier. Dans certains chapitres, l'auteur parle moins du recensement en tant que tel que de concepts ou de problèmes qui lui sont associés. Le cinquième chapitre passe ainsi beaucoup plus de temps à explorer la manière dont la question de la limitation des naissances s'est développée, dans le contexte intellectuel de « l'explosion démographique » de l'après-guerre, et le contexte politique de la Guerre froide et du développement de l'ONU, qu'à discuter des évolutions du recensement proprement dit. On retrouve ce problème à plusieurs endroits dans le livre. Cela ne gêne pas la lecture, bien au contraire, mais en dilue quelque peu le propos central.

Pour conclure, au-delà de la qualité du contenu du point de vue académique, il me semble qu'il est nécessaire de noter l'élégance de l'écriture et les qualités de conteur de l'auteur. Ce livre n'est que l'un des plus récents sur le recensement, mais il se distingue incontestablement par la facilité avec laquelle on est plongé dans l'histoire de cet instrument rarement vanté pour son lustre. Chaque chapitre ouvre sur une histoire différente, une mise en contexte des enjeux présentés dans le chapitre, raconté avec un incontestable talent. En transportant le lecteur de la mission spectaculaire de la Royal Air Force visant à détruire des registres hollandais utilisés par les nazis, à la visite de l'auteur du petit musée sur Malthus dans sa modeste ville natale, ou encore ses entretiens avec un énergique ancien directeur du recensement sud-africain, Whitby signe là un premier livre remarquablement riche et accessible.

Quentin Wallut Doctorant en Science politique à l'UQAM wallut.quentin@uqam.ca 


\section{BIBLIOGRAPHIE}

ANDERSON, B. 1983. Imagined Communities : Reflections on the Origin and Spread of Nationalism. London, New York, Verso.

EMIGH, R. J., D. RILEY et P. AHMED. 2016a. Antecedents of Censuses from Medieval to Nation States: How Societies and States Count. New York, Springer.

- 2016b. Changes in Censuses from Imperialist to Welfare States: How Societies and States Count. New York, Springer.

LEIBLER, A. 2004. "Statisticians' Ambition : Governmentality, Modernity and National Legibility », Israel Studies, 9, $2: 121-149$.

LEIBLER, A. et D. BRESLAU. 2005. "The uncounted : Citizenship and exclusion in the Israeli census of 1948 », Ethnic and Racial Studies, 28, 5 : 880-902.

SCHOR, P. 2009. Compter et classer - Histoire des recensements américains. École des Hautes Études en Science Sociales, Paris, EHESS.

SELTZER, W. et M. ANDERSON. 2001. "The Dark Side of Numbers : The Role of Population Data Systems in Human Rights Abuses », Social Research, 68, 2 : 481-513.

THORVALDSEN, G. 2017. Censuses and Census Takers : A Global History. London, Routledge.

VENTRESCA, M. J. 1995. "When states count : Institutional and political dynamics in modern census establishment, 1800-1993 ", (Thèse de doctorat), Stanford University, États-Unis. 